\title{
A randomized controlled trial to evaluate the efficacy of metacognitive training for people with schizophrenia applied by mental health nurses: Study protocol
}

\author{
Lara Manuela Guedes de Pinho RMN, MSc, PhD, Researcher, Adjunct Professor, Doctorate \\ Integrated Member, PhD in Psychology, Master in Clinical and Health Psychology, Mental
} Health Nurse, PhD Student in Nurses and Health ${ }^{1,2,3,4}$ (D) | Carlos Alberto da Cruz Sequeira RMN, MSc, PhD, Coordinating Professor, Coordinator Research Group, PhD in Science in Nursing, Master in Public Health, Mental Health Nurse H,5 $^{3,5}$ | Francisco Miguel Correia Sampaio RMN, MSc, PhD, Assistant Professor, Doctorate Integrated Member, Postdoctoral Researcher, PhD in Nursing Science, Master in Psychiatric, Mental Health Nurse $^{3,6,7}$ (D) | Nuno Barbosa Rocha MSc, PhD, Professor, Vice-President, Vice-Director, PhD in Psychology, Master in Psychology, Registered Psychologist and Occupational Therapist ${ }^{8,9}$ | Carmen Ferre-Grau RMN, MSc, PhD, Titular Professor, Doctorate Programme Coordinator, PhD in Psychology, Master in Public Health, Mental Health Nurse ${ }^{10,11}$

\footnotetext{
${ }^{1}$ Nursing School of São João de Deus, University of Évora, Évora, Portugal

${ }^{2}$ Health School of the Portalegre Polytechnic Institute, Portalegre, Portugal

${ }^{3}$ NURSID group of Center for Health Technology and Services Research (CINTESIS), Porto, Portugal

${ }^{4}$ Universitat Rovira y Virgili, Tarragona, Spain

${ }^{5}$ School of Nursing of Porto, Porto, Portugal

${ }^{6}$ Faculty of Health Sciences, University

Fernando Pessoa, Porto, Portugal

${ }^{7}$ Faculty of Medicine, University of Porto,

Porto, Portugal

${ }^{8}$ School of Health, Polytechnic of Porto, Porto, Portugal

${ }^{9}$ Center for Rehabilitation Research, Porto, Portugal

${ }^{10}$ Universitat Rovira y Virgili, Tarragona, Spain

${ }^{11}$ Nurse and Health URV, Tarragona, Spain

\begin{abstract}
Aim: To evaluate the efficacy of the Portuguese version of the metacognitive training (MCT) programme for schizophrenia and its effects on psychotic symptoms, insight into the disorder and functionality.

Design: This is a randomized controlled trial that will be undertaken in six psychiatric institutions in Portugal.

Methods: This study was approved in March 2019. The sample will consist of people with schizophrenia. The evaluation instruments will include sociodemographic and clinical questionnaires, the Psychotic Symptom Rating Scales, the Beck Cognitive Insight Scale, the World Health Disability Assessment Schedule, and the Personal and Social Performance Scale, applied to both groups at three different times. In the experimental group, the eight MCT modules will be applied over 4 weeks.

Discussion: It is expected that at the end of the programme, the experimental group will have reduced severity of psychotic symptoms and improved insight into the disease and functionality.

Impact: Schizophrenia is a severe mental disorder that, in most cases, leads to the deterioration of cognitive and social functioning as a result of psychotic symptoms.
\end{abstract}


Funding information

CINTESIS, R\&D Unit, Grant/Award Number: UID/IC/4255/2019; Portuguese Society of Mental Health Nursing (ASPESM), Grant/Award Number: POCI-01-0145FEDER-007746; Fundação para a Ciência e a Tecnologia, Grant/Award Number: UID/IC/4255/2013; FEDER, Grant/Award Number: POCI-01-0145-FEDER-007746
Metacognitive training for schizophrenia has been used in several countries, but its efficacy remains unclear. It is a type of programme that consists of changing the cognitive infrastructure of delusions.

\section{KEYWORDS}

functionality, insight, mental health nurses, metacognitive training, psychotic symptoms, randomized controlled trial, schizophrenia

\section{1 | INTRODUCTION}

Mental diseases are responsible for more than $12 \%$ of the global burden of disease worldwide and $23 \%$ in developed countries. Five of the top ten causes of long-term disability and psychosocial dependence are mental diseases: unipolar depression (11.8\%), alcohol problems (3.3\%), schizophrenia (2.8\%), bipolar disorders (2.4\%), and dementia (1.6\%); (Xavier, Baptista, Mendes, Magalhães, \& Caldasde-Almeida, 2013 quoted in Carvalho, 2013).

Schizophrenia is a severe mental disorder characterized by psychotic symptoms (positive and negative symptoms). Delusions (thought changes), hallucinations (perception changes), and disorganization of thinking and behaviour are considered positive symptoms. The most frequent negative symptoms are avolition, decrease in emotional expression, alogia, anhedonia, and social isolation (American Psychiatric Association, 2014).

Delusions and hallucinations are two psychotic symptoms that characterize schizophrenia. Over time, this disease usually causes cognitive and social dysfunction. In many cases, treatment of schizophrenia with medication alone is still a reality. In addition, there are not enough resources for this condition in the community and the implementation of psychotherapeutic strategies for psychosocial rehabilitation is scarce. Thus, it is urgent that we develop more effective care management strategies in response to this problem.

\section{1 | Background}

In most cases, the exclusive treatment of schizophrenia with medication is not very effective and is insufficient for patient rehabilitation. Additionally, poor insight and low adherence to treatment require the implementation of psychotherapies that complement treatment with neuroleptics. In this sense, cognitive-behavioural therapy approaches and family interventions have been shown to be a fundamental complement to psychopharmacology (Pfammatter, Andres \& Brenner, 2012; Townsend, 2011; Wykes, Steel, Everitt, \& Tarrier, 2008).

Metacognitive training (MCT) for psychosis is a cognitive-behavioural therapy registered by Steffen Moritz in 2007. Some recent studies have demonstrated that MCT is beneficial for people with schizophrenia and lowers the severity of delusions, as demonstrated by recent meta-analyses (Eichner \& Berna, 2016; Jiang, Zhang, Zhipei, Wei, \& Chunbo, 2015; Liu, Tang, Hung, Tsai, \& Lin, 2018; van Oosterhout et al., 2016). However, these meta-analyses showed different results. While the results of Liu et al. (2018) and Eichner and Berna (2016) demonstrated that after MCT application, the severity of delusion decreased immediately, meta-analyses by Jiang el al. (2015) and van Oosterhout et al. (2016) showed that delusions decreased only over time. Furthermore, the positive effect of MCT at 6 months post-intervention was demonstrated only in the most recent meta-analysis (Liu et al., 2018). Thus, more studies are necessary to prove the efficacy of MCT.

The four meta-analyses presented studied the effects of MCT in 12 countries: Australia, England, Finland, France, Germany, Hong Kong, India, Ireland, the Netherlands, Poland, Portugal, and Switzerland. In Portugal, Rocha and Queirós (2013) studied the preliminary efficacy of Metacognitive and Social Cognition Training (MSCT) in schizophrenia with 18 sessions. In this study, MSCT had no effect on positive symptoms, contrary to expectations (Rocha \& Queirós, 2013). MCT has not yet been validated in Portugal and studies are needed to prove its efficacy in the Portuguese population. Therefore, to evaluate the efficacy of this programme in people with schizophrenia, a randomized controlled trial will be conducted using the protocol presented in this article. MCT will be applied by mental health nurses. The research question of this study is as follows: 'Is metacognitive training in people with schizophrenia effective in reducing psychotic symptoms and improving insight into the disease and functionality?'

\section{THE STUDY}

\subsection{Aim}

The aim of this study is to evaluate the efficacy of a metacognitive group training in people with schizophrenia and its effects on psychotic symptoms, insight into the disorder and functionality. The hypotheses to be validated in this trial are as follows:

- Compared with the control participants, the people with schizophrenia who join the experimental group and participate in the MCT programme will have reduced severity of psychotic symptoms, will present better insight into their disease, and will have improved functionality on completion of the programme;

- In the MCT programme participants, psychotic symptoms will be reduced at the end of the programme and at follow-up (3 months later) and their consciousness of the disease and functionality will improve. 


\section{2 | Design/methodology}

\subsection{1 | Study design}

The MCT programme groups were designed for a randomized controlled trial. The sample will be selected by a probabilistic method and participants will be randomly distributed into one of two groups (experimental or control). Taking into account the evolution of schizophrenia and the possible influence of sociodemographic and clinical characteristics, the stratified random sampling method will be applied.

All procedures shall be in accordance with the CONSORT (Consolidated Standards of Reporting Trials) guidelines and follow four phases: enrolment, intervention allocation, follow-up, and data analysis. The follow-up will be undertaken 3 months after the programme. The trial has been registered at ClinicalTrials.gov (ID NCT03891186).

\subsection{2 | Participants}

The population of this study will be composed of people with schizophrenia who attend psychosocial rehabilitation programmes or socio-occupational forums in psychiatric institutions in Portugal.

Inclusion criteria

Age between 18-65 years; diagnosis of schizophrenia evaluated by a psychiatric assistant; and no changes in neuroleptic medication within for 4 months before the programme.

\section{Exclusion criteria}

Substance dependence; very severe psychotic symptoms that impede understanding of the objectives of the sessions; and changes in neuroleptic medication within 4 months before the programme.

\subsection{3 | Randomization and blinding}

Eligible participants will be recruited by the researchers in collaboration with a multidisciplinary team from each institution. A baseline assessment will be carried out and the instruments will be applied after informed consent from the participants. Participants will be randomly allocated to either the MCT (experimental group) or the control group. The control group will not participate in the MCT programme. In both groups, treatment will be maintained as usual. Participants will be stratified by educational level, duration of mental disorder, and type of treatment. All participants will be re-assessed at the end of the programme and 3 months after completion. The programme will be applied over 4 weeks (two sessions per week) by a mental health nurse at the institution. The instruments will be applied by the same researcher before and after the programme and at follow-up. This researcher will not know to which group the participants belong. Thus, the researcher that applies the instruments will be blinded (Figure 1).

\subsection{4 | Sample size determination}

Assuming an effect size like those of previous studies (Lam et al., 2015; Moritz et al., 2013) and considering $\alpha=0.05$ and power $=0.80$, a total sample size of 36 (18 participants per condition) could be considered to detect an effect in the primary outcome (partial eta square $=0.04)$. Sample size calculation was performed with G*Power (Erdfelder, Faul, \& Buchner, 1996). Considering a dropout of $10 \%$, we aimed to include 40 participants.

\subsection{5 | Study intervention}

\section{Treatment as usual}

All participants will continue to receive treatment as usual (TAU) at their institution. TAU consists of psychiatric and psychosocial treatment by a multidisciplinary team, including mental health psychiatric nurses, psychiatrists, psychologists, social workers, and occupational therapists. TAU includes community treatment, day hospital, and hospital admission and involves antipsychotic medication, psychosocial rehabilitation, socialization groups, and psychoeducational and therapy groups. In this way, procedural ethics will be maintained. The control group will not participate in the MCT programme.

\section{Metacognitive training for psychosis}

Metacognitive training is a cognitive approach to the treatment of positive symptoms in psychosis that has been translated into 37 languages. MCT was developed by Moritz and colleagues (Moritz \& Woodward, 2007; Moritz, Vitzthum, Randjbar, Veckenstedt, \& Woodward, 2010; Moritz, Woodward, \& Rocha, 2010) and all information about this programme is available at https://clinical-neuropsychology.de/metacognitive_training-psych osis/. The most recent Portuguese version was concluded in 2017 (Portuguese version 6.3; Moritz, Woodward, Hauschildt \& Rocha, 2017). Specific terms were adapted to Portugal, but the examples were kept the same for consistency across countries. However, the intervention and examples can be tailored by the therapist to meet specific group needs.

Metacognitive training is composed of eight modules referring to common cognitive issues and biases in solving problems in schizophrenia. The topics of MCT include the following: attribution blaming and taking credit (module 1), jumping to conclusions (modules 2 $\& 7$ ), changing beliefs (module 3 ), deficits in theory of mind and social cognition (modules 4 and 6), overconfidence in (memory) errors (module 5), and depression and low self-esteem (module 8).

The aim of the sessions is to convey knowledge about cognitive distortions, such as false beliefs and delusions and helping patients to reflect critically with exercises and acquire new problem-solving strategies. Each session lasts 45-60 min and follows a protocol defined in the 'Metacognitive Training for Psychosis (MCT)' manual. The materials used in MCT are PowerPoint slides, videos, homework exercises, and yellow and red cards. These materials are available at www.uke.de/mkt. 
FIGURE 1 Study schedule

\begin{tabular}{|c|c|c|c|c|c|}
\hline \multirow[b]{3}{*}{ TIMEPOINT** } & \multicolumn{5}{|c|}{ STUDY PERIOD } \\
\hline & \multirow{2}{*}{$\begin{array}{c}\text { Enrolment } \\
-t_{1}\end{array}$} & \multirow{2}{*}{$\begin{array}{c}\text { Allocation } \\
0\end{array}$} & \multicolumn{2}{|c|}{$\begin{array}{c}\text { Post- } \\
\text { allocation }\end{array}$} & \multirow{2}{*}{$\begin{array}{r}\text { Close-out } \\
T_{3}\end{array}$} \\
\hline & & & $t_{1}$ & $t_{2}$ & \\
\hline \multicolumn{6}{|l|}{ ENROLMENT: } \\
\hline Eligibility screen & $x$ & & & & \\
\hline \multirow{2}{*}{$\begin{array}{r}\text { Informed consent } \\
\text { [List other } \\
\text { procedures] }\end{array}$} & $x$ & & & & \\
\hline & $x$ & & & & \\
\hline Allocation & & $x$ & & & \\
\hline \multicolumn{6}{|l|}{ INTERVENTIONS: } \\
\hline \\
\hline \multirow{2}{*}{\multicolumn{6}{|c|}{$\begin{array}{r}\text { [Metacognitive } \\
\text { training for } \\
\text { Psicose }(M C T)]\end{array}$}} \\
\hline & & & & & \\
\hline \multicolumn{6}{|l|}{$\begin{array}{r}\text { [List other study } \\
\text { groups] }\end{array}$} \\
\hline \multicolumn{6}{|l|}{ ASSESSMENTS: } \\
\hline \multirow{3}{*}{$\begin{array}{r}\text { [] } \\
\text { [PSYRATS, BCIS, } \\
\text { WHODAS 2.0, PSP] } \\
\text { [Subjective } \\
\text { appraisal of the } \\
\text { interventions] }\end{array}$} & $x$ & & & & \\
\hline & & $x$ & & $x$ & $x$ \\
\hline & & & & $x$ & $x$ \\
\hline
\end{tabular}

The MCT for Schizophrenia programme will be applied to the experimental group by mental health and psychiatry specialist nurses with professional experience in the care of people with schizophrenia. A practical course on $\mathrm{MCT}$ was implemented and all mental health and psychiatry nurses received training on the application of the MCT programme. It will be applied in two sessions per week for a total of eight sessions.

Metacognitive training will be applied face-to-face in groups in a quiet room of the psychiatric institution to which the group belongs. If a participant's neuroleptic medication is changed during the course of the programme, they will be excluded from the study. To improve adherence to $\mathrm{MCT}$, the sessions will be applied during the period of usual treatment.

\subsection{6 | Data collection and outcome measures}

The instruments will be applied to all participants in the study through an interview at the three assessment times. To characterize the sample, sociodemographic, and clinical data will be collected (age, gender, marital status, cohabitation, educational level, professional/employment status, duration of mental disorder, number of psychiatric hospitalizations, medication, type of treatment, and substance use). These data will be collected before the beginning of the programme for all participants.

\section{PSYRATS - Psychotic symptom rating scales}

This instrument is a structured interview that provides a detailed measurement of delusions and hallucinations. The PSYRATS have 17 items and two subscales: one subscale assesses delusions (6 items) and the other assesses hallucinations (11 items). Each item has a classification of five points (0-4). It was developed by Haddock, McCarron, Tarrier, and Faragher (1999) (validated for the Portuguese population by Telles-Correia et al. (2017)).

\section{BCIS - Beck cognitive insight scale}

This scale has 15 items and is a self-response questionnaire developed by Beck, Baruch, Balter, Steer, and Warman (2004) that evaluates consciousness of the illness. (It is in the process of validation for the Portuguese population by the researchers of this study.) 


\section{WHODAS 2.0 - World health disability assessment schedule}

This instrument evaluates functionality levels and was developed by the WHO. It has 12 items and is a self-response questionnaire (validated for the Portuguese population by Moreira, Alvarelhão, Silva, Costa, and Queirós (2015)).

\section{PSP - Personal and social performance scale}

This scale was developed by Morosini, Magliano, Brambilla, Ugolini, and Pioli (2000) and assesses patients' social functioning with regard to four main areas: socially useful activities, personal and social relationships, self-care, and disturbing and aggressive behaviours (validated for the Portuguese population by Brissos et al., 2012).

The PSYRATS, BCIS, WHODAS 2.0, and PSP will be applied to both groups before the first session of MCT and a week after the eight sessions. Three months later, these instruments will be reapplied for follow-up evaluation in both the groups. These evaluations will be conducted by a different person than the one who applied the MCT programme to avoid bias and they will be applied in person.

\section{Subjective appraisal of the interventions}

In the pilot study, at the end of the MCT for Schizophrenia and at follow-up, an anonymous questionnaire will be conducted to assess acceptance, feasibility, and subjective efficacy of the interventions. This instrument was applied in previous trials (Moritz, Kerstan, et al., 2011; Moritz et al., 2013; Moritz, Veckenstedt, Randjbar, Vitzthum, \& Woodward, 2011; Moritz \& Woodward, 2007) and it will be completed only by the experimental group. The outcome measures are listed in Table 1.

\subsection{7 | Data analysis}

The data will be analysed using Statistical Package for Social Sciences software (SPSS ${ }^{\circledR}$ ) version 24.0 for Windows. Descriptive analysis will be used to characterize the sample. To compare the results in both groups and to compare the results of the PSYRATS, BCIS, WHODAS, and PSP at the three application times, statistical inference will be used.

\subsection{Ethical considerations and dissemination}

Approval was obtained from all the institutions' ethics committees. All participants will be informed of the study objectives, methodology, benefits, and possible risks. They will sign the written informed consent required for this study. Participant confidentiality will be maintained throughout all study procedures. Participants will be informed that they may withdraw their participation at any time without penalty. Only members of the research team and health professionals that care for the participants have access to participant data. These data will be destroyed at the end of the study.

Any modifications to the protocol that may have an impact on the implementation of the study, for example, changes in study design, study objectives, and study procedures, will be revised by independent researchers and will be communicated to the ethical committees. The results of the study will be disseminated through oral communications in congresses, publication in journals and posters.

\section{4 | Validity and reliability/rigour}

Variables such as educational level, duration of mental disorder, and type of treatment may influence the results of this study. The proposed stratified random sampling method in this study is intended to minimize confounding bias.

\section{3 | DISCUSSION}

In some countries, previous studies have proved the efficacy of MCT and its ability to reduce positive symptoms (Aghotor, Pfueller, Moritz, Weisbrod, \& Roesch-Ely, 2010; Andreou et al., 2017; Briki et al., 2014; Favrod et al., 2014; Kumar et al., 2010; Moritz et al., 2013; So et al., 2015). However, other studies did not find this efficacy in reducing positive symptoms (Gaweda, Krezołek, Olbrys, Turska, \& Kokoszka, 2015; van Oosterhout et al., 2014). More studies are needed to accurately prove the efficacy of MCT.

With respect to cognitive insight, a randomized control trial with a sample of 80 people with schizophrenia showed significant improvements in cognitive insight in the experimental group (Lam et al., 2015). However, in another randomized control trial with a sample of 154 people with schizophrenia, MCT was not more efficacious in terms of cognitive insight (van Oosterhout et al., 2014). Both studies used the BCIS to evaluate cognitive insight. The efficacy of the MCT programme for people with schizophrenia has not yet been studied

TABLE 1 Outcomes measures and data collection

\begin{tabular}{|c|c|c|c|c|}
\hline \multirow[b]{2}{*}{ Instrument } & \multirow[b]{2}{*}{ Applied to: } & \multicolumn{3}{|c|}{ Data collection time } \\
\hline & & $\begin{array}{l}\text { M1 (initial } \\
\text { evaluation) }\end{array}$ & $\begin{array}{l}\text { M2 (final } \\
\text { evaluation) }\end{array}$ & $\begin{array}{l}\text { M3 (follow-up } \\
\text { at } 3 \text { months) }\end{array}$ \\
\hline Sociodemographic and clinical data & Experimental group and control group & $X$ & & \\
\hline PSYRATS & Experimental group and control group & $X$ & $X$ & $X$ \\
\hline $\mathrm{BCIS}$ & Experimental group and control group & $X$ & $X$ & $X$ \\
\hline Subjective appraisal of the interventions & Experimental group of the pilot study & & X & X \\
\hline
\end{tabular}


in the Portuguese population and thus the development of the present trial is paramount.

The most recent meta-analysis of randomized control trials that used the MCT programme for people with schizophrenia concluded that for MCT to be successful in the future, a better understanding of the mechanism and factors that improve the efficacy of MCT may be helpful (Liu et al., 2018). The greatest challenges expected in the development of this trial are related to the potential of people with schizophrenia to not consent to participate in the research due to the low adherence to treatment and poor insight into disease characteristic of people with this disease. This could influence the sample size. To minimize this potential constraint, six psychiatric institutions were chosen to collect data.

It is expected that the results of this trial will validate the Portuguese version of MCT and prove its efficacy in reducing the severity of psychotic symptoms, and in improving consciousness of the illness and functioning in people with schizophrenia. The results of this trial are expected to allow future implementation of the MCT programme by mental health and psychiatry specialist nurses and other mental health professionals in various mental health institutions.

\section{1 | Limitations}

In this study, the setting is confined to six institutions to Portugal, which will limit the generalizability of the results to other countries.

\section{4 | CONCLUSION}

It has been proven that the best treatment for schizophrenia is the combination of psychopharmacology and cognitive-behavioural therapy. MCT intends to contribute to the psychosocial rehabilitation of people with schizophrenia used by mental health nurses or other mental health professionals. The trial illustrated in this protocol intends to assess the efficacy of MCT in the Portuguese population.

\section{ACKNOWLEDGEMENTS}

The authors would like to thank all collaborators of the psychiatric organizations where the protocol will be applied. Thank Ana Luisa, André Louro, Bruno Santos, Catarina Silva, José João Silva, Maria da Luz Rocha, Mónica Murraceira, Paulo Seabra, Raquel Martins, Rodrigo Mendes and Sara Serralheiro.

\section{CONFLICT OF INTEREST}

No conflict of interest has been declared by the author(s).

\section{AUTHORS' CONTRIBUTIONS}

LGP initiated the study design; CS and CFG initiated the study design and revised the study; FS provided statistical expertise in clinical trial design; and NR provided theoretical, practical, and research knowledge about metacognitive training. All authors contributed to refinement of the study protocol and approved the final manuscript.

\section{ORCID}

Lara Manuela Guedes de Pinho (iD https://orcid. org/0000-0003-1174-0744

Carlos Alberto da Cruz Sequeira (iD https://orcid. org/0000-0002-5620-3478

Francisco Miguel Correia Sampaio (iD https://orcid. org/0000-0002-9245-256X

\section{REFERENCES}

Aghotor, J., Pfueller, U., Moritz, S., Weisbrod, M., \& Roesch-Ely, D. (2010). Metacognitive training for patients with schizophrenia (MCT): Feasibility and preliminary evidence for its efficacy. Journal of Behavior Therapy and Experimental Psychiatry, 41(3), 207-211. https:// doi.org/10.1016/j.jbtep.2010.01.004

American Psychiatric Association. (2014). DSM-5 Manual de diagnóstico e estatística das perturbações mentais ( $5^{\mathrm{a}}$ ed.). Lisboa: Climepsi Editores.

Andreou, C., Wittekind, C. E., Fieker, M., Heitz, U., Veckenstedt, R., Bohn, F., \& Moritz, S. (2017). Individualized metacognitive therapy for delusions: A randomized controlled rater-blind study. Journal of Behavior Therapy and Experimental Psychiatry, 56, 144-151. https:// doi.org/10.1016/j.jbtep.2016.11.013

Beck, A. T., Baruch, E., Balter, J. M., Steer, R. A., \& Warman, D. M. (2004). A new instrument for measuring insight: The Beck Cognitive Insight Scale. Schizophrenia Research, 68(2-3), 319-329. https://doi. org/10.1016/S0920-9964(03)00189-0

Briki, M., Monnin, J., Haffen, E., Sechter, D., Favrod, J., Netillard, C., ... Vandel, P. (2014). Metacognitive training for schizophrenia: A multicentre randomised controlled trial. Schizophrenia Research, 157(1), 99-106. https://doi.org/10.1016/j.schres.2014.06.005

Brissos, S., Palhavã, F., Marques, J. G., Mexia, S., Carmo, A. L., Carvalho, M., ... Figueira, M. L. (2012). The Portuguese version of the Personal and Social Performance Scale (PSP): Reliability, validity and relationship with cognitive measures in hospitalized and community schizophrenia patients. Social Psychiatry and Psychiatric Epidemiology, 47(7), 1077-1086. https://doi.org/10.1007/ s00127-011-0412-6

Carvalho, A. (2013). Portugal. Saúde mental em números - 2013 - Programa nacional para a saúde mental. Lisboa: Direcção-Geral da Saúde.

Eichner, C., \& Berna, F. (2016). Acceptance and efficacy of metacognitive training $(\mathrm{MCT})$ on positive symptoms and delusions in patients with schizophrenia: A meta-analysis taking into account important moderators. Schizophrenia Bulletin, 42(4), 952-962. https://doi. org/10.1093/schbul/sbv225

Erdfelder, E., Faul, F., \& Buchner, A. (1996). GPOWER: A general power analysis program. Behaviour Research Methods Instruments and Computers, 28, 1-11. https://doi.org/10.3758/BF03203630

Favrod, J., Rexhaj, S., Bardy, S., Ferrari, P., Hayoz, C., Moritz, S., ... Bonsack, C. (2014). Sustained antipsychotic effect of metacognitive training in psychosis: A randomized-controlled study. European Psychiatry, 29(5), 275-281. https://doi.org/10.1016/j. eurpsy.2013.08.003

Gaweda, L., Krezołek, M., Olbrys, J., Turska, A., \& Kokoszka, A. (2015). Decreasing self-reported cognitive biases and increasing clinical insight through meta-cognitive training in patients with chronic schizophrenia. Journal of Behavioral Therapy and Experimental Psychiatry, 48, 98-104. https://doi.org/10.1016/j.jbtep.2015.02.002 
Haddock, G., McCarron, J., Tarrier, N., \& Faragher, E. B. (1999). Scales to measure dimensions of hallucinations and delusions: The psychotic symptom rating scales (PSYRATS). Psychological Medicine, 29(4), 879-889. https://doi.org/10.1017/s0033291799008661

Jiang, J., Zhang, L., Zhipei, Z. H. U., Wei, L. I., \& Chunbo, L. I. (2015). Metacognitive training for schizophrenia: A systematic review. Shanghai Archives of Psychiatry, 27(3), 149. https://doi.org/10.11919/ j.issn.1002-0829.215065

Kumar, D., Zia UI Haq, M., Dubey, I., Dotivala, K. N., Veqar Siddiqui, S., Prakash, R., ... Nizamie, S. H. (2010). Effect of meta-cognitive training in the reduction of positive symptoms in schizophrenia. European Journal of Psychotherapy and Counselling, 12(2), 149-158. https://doi. org/10.1080/13642537.2010.488875

Lam, K. C. K., Ho, C. P. S., Wa, J. C., Chan, S. M. Y., Yam, K. K. N., Yeung, O. S. F., ... Balzan, R. P. (2015). Metacognitive training (MCT) for schizophrenia improves cognitive insight: A randomized controlled trial in a Chinese sample with schizophrenia spectrum disorders. Behaviour Research and Therapy, 64, 38-42. https://doi.org/10.1016/j. brat.2014.11.008

Liu, Y. C., Tang, C. C., Hung, T. T., Tsai, P. C., \& Lin, M. F. (2018). The efficacy of metacognitive training for delusions in patients with schizophrenia: A meta-analysis of randomized controlled trials informs evidence-based practice. Worldviews on Evidence-Based Nursing, 15(2), 130-139. https://doi.org/10.1111/wvn.12282

Moreira, A., Alvarelhão, J., Silva, A. G., Costa, R., \& Queirós, A. (2015). Tradução e validação para português do WHODAS 2.0: 12 itens em pessoas com 55 ou mais anos. Revista Portuguesa de Saúde Pública, 33(2), 179-182.

Moritz, S., Kerstan, A., Veckenstedt, R., Randjbar, S., Vitzthum, F., Schmidt, C., ... Woodward, T. S. (2011). Further evidence for the efficacy of a metacognitive group training in schizophrenia. Behaviour Research and Therapy, 49(3), 151-157. https://doi.org/10.1016/j. brat.2010.11.010

Moritz, S., Veckenstedt, R., Bohn, F., Hottenrott, B., Scheu, F., Randjbar, S., ... Roesch-Ely, D. (2013). Complementary group Metacognitive Training (MCT) reduces delusional ideation in schizophrenia. Schizophrenia Research, 151(1), 61-69. https://doi.org/10.1016/j. schres.2013.10.007

Moritz, S., Veckenstedt, R., Randjbar, S., Vitzthum, F., \& Woodward, T. (2011). Antipsychotic treatment beyond antipsychotics: Metacognitive intervention for schizophrenia patients improves delusional symptoms. Psychological Medicine, 41(9), 1823-1832. https:// doi.org/10.1017/S0033291710002618

Moritz, S., Vitzthum, F., Randjbar, S., Veckenstedt, R., \& Woodward, T. (2010). Detecting and defusing cognitive traps: Metacognitive intervention in schizophrenia. Current Opinion in Psychiatry, 23, 561-569. https://doi.org/10.1097/YCO.0b013e32833d16a8

Moritz, S., \& Woodward, T. S. (2007). Metacognitive training in schizophrenia: From basic research to knowledge translation and intervention. Current Opinion in Psychiatry, 20(6), 619-625. https://doi. org/10.1097/YCO.0b013e3282f0b8ed

Moritz, S., Woodward, T. S., Hauschildt, M., \& Rocha, N. B. (2017).Treino Metacognitivo para Psicose (MCT), versãoportuguesa 6.3. (tradução e adaptação portuguesa por Nuno Rocha, Cristina Queirós, Susana Rocha \& Susana Pereira), available in www.uke.de/mkt.
Moritz, S., Woodward, T., \& Rocha, N. (2010). Treino Metacognitivo para pacientes com Esquizofrenia (TMC)-Manual. Hamburg: VanHam Campus Press.

Morosini, P. L., Magliano, L., Brambilla, L., Ugolini, S., \& Pioli, R. (2000). Development, reliability and acceptability of a new version of the DSM-IV Social and Occupational Functioning Assessment Scale (SOFAS) to assess routine social functioning. Acta Psychiatrica Scandinavica, 101, 323-329.

Pfammatter, M.,Andres, K., \&Brenner,H.(2012).ManualdePsicoeducação e Gestão da Doença naesquizofrenia. EdiçãoEncontrar-se.

Rocha, N. B., \& Queirós, C. (2013). Metacognitive and social cognition training (MSCT) in schizophrenia: A preliminary efficacy study. Schizophrenia Research, 150(1), 64-68. https://doi.org/10.1016/j. schres.2013.07.057

So, S. H.-W., Chan, A. P., Chong, C. S.-Y., Wong, M. H.-M., Lo, W. T.-L., Chung, D. W.-S., \& Chan, S. S. (2015). Metacognitive training for delusions (MCTd): Effectiveness on data-gathering and belief flexibility in a Chinese sample. Frontiers in Psychology, 6(730), 1-13. https://doi. org/10.3389/fpsyg.2015.00730

Telles-Correia, D., Barbosa-Rocha, N., Gama-Marques, J., Moreira, A. L., Alves-Moreira, C., \& Saraiva, S. ... Haddock, G. (2017). Vlidación de la versión en portugués de la Escala de Evaluación de Síntomas Psicóticos (PSYRATS). Actas Espanolas de Psiquiatria, 45(2), 56-61.

Townsend, M. C. (2011). Enfermagem em Saúde Mental e Psiquiátrica (6a ed.). Loures: Lusociência.

van Oosterhout, B., Krabbendam, L., De Boer, K., Ferwerda, J., van der Helm, M., Stant, A., \& van der Gaag, M. (2014). Metacognitive group training for schizophrenia spectrum patients with delusions: A randomized controlled trial. Psychological Medicine, 44(14), 3025-3035. https://doi.org/10.1017/S0033291714000555

van Oosterhout, B., Smit, F., Krabbendam, L., Castelein, S., Staring, A., \& van der Gaag, M. (2016). Metacognitive training for schizophrenia spectrum patients: A meta-analysis on outcome studies. Psychological Medicine, 46(1), 47-57. https://doi.org/10.1017/S0033 291715001105

Wykes, T., Steel, C., Everitt, B., \& Tarrier, N. (2008). Cognitive behavior therapy for schizophrenia: Effect sizes, clinical models and methodological rigor. Schizophrenia Bulletin, 34, 523-537. https://doi. org/10.1093/schbul/sbm114

Xavier, M., Baptista, H., Mendes, J. M., Magalhães, P., \& Caldas-deAlmeida, J. M. (2013). Implementing the World Mental Health Survey Initiative in Portugal - rationale, design and fieldwork procedures. International Journal of Mental Health Systems, 7, 19.

How to cite this article: de Pinho LMG, Sequeira CAdaC, Sampaio FMC, Rocha NB, Ferre-Grau C. A randomized controlled trial to evaluate the efficacy of metacognitive training for people with schizophrenia applied by mental health nurses: Study protocol. J Adv Nurs. 2020;76:356-363. https://doi.org/10.1111/jan.14240 
The Journal of Advanced Nursing (JAN) is an international, peer-reviewed, scientific journal. JAN contributes to the advancement of evidence-based nursing, midwifery and health care by disseminating high quality research and scholarship of contemporary relevance and with potential to advance knowledge for practice, education, management or policy. JAN publishes research reviews, original research reports and methodological and theoretical papers.

For further information, please visit JAN on the Wiley Online Library website: www.wileyonlinelibrary.com/journal/jan

Reasons to publish your work in JAN:

- High-impact forum: the world's most cited nursing journal, with an Impact Factor of 1998 - ranked 12/114 in the 2016 ISI Journal Citation Reports $\odot$ (Nursing (Social Science)).

- Most read nursing journal in the world: over 3 million articles downloaded online per year and accessible in over 10,000 libraries worldwide (including over 3,500 in developing countries with free or low cost access).

- Fast and easy online submission: online submission at http://mc.manuscriptcentral.com/jan.

- Positive publishing experience: rapid double-blind peer review with constructive feedback.

- Rapid online publication in five weeks: average time from final manuscript arriving in production to online publication.

- Online Open: the option to pay to make your article freely and openly accessible to non-subscribers upon publication on Wiley Online Library, as well as the option to deposit the article in your own or your funding agency's preferred archive (e.g. PubMed). 